\title{
Load Based Cell Selection Algorithm for Digital Mobile Radio
}

\author{
Simay Yılmaz, Berna Özbek \\ Department of Electrical and Electronics Engineering \\ Izmir Institute of Technology \\ Izmir, Turkey \\ \{simayyilmaz,bernaozbek\}@iyte.edu.tr
}

\author{
Murat Taş, Erinç Deniz Bardak \\ ASELSAN A.Ş. \\ Ankara, Turkey \\ $\{$ mtas,edbardak\}@aselsan.com.tr
}

\begin{abstract}
${ }^{1}$ Abstract-Digital mobile radio is one of a digital radio standard for Professional Mobile Radio and it is commonly used for emergency services. The cell selection is very important for digital mobile radio based systems to improve system performance in terms of delay and bit error rate. In this work, we propose an efficient cell selection algorithm for Digital Mobile Radio. In the proposed algorithm, each user selects the base station according to the proposed utility value determined based on both cell load and signal-to-interference-noise ratio. The goal of the proposed algorithm is to balance the distribution of the users among the cells to reduce the waiting time for connection while establishing reliable transmission link. We illustrate the performance results for different scenarios and applications in terms of cell load, signal-to-interference-noise ratio and bit error rate.
\end{abstract}

Index Terms-Cell Selection, Digital Mobile Radio, Professional Mobile Radio.

\section{INTRODUCTION}

Professional Mobile Radio (PMR) is widely used for emergency services by providing facilities for closed user groups, group call and push-to-talk. It has short call set-up times compared with cellular systems while referring to the two-way radio communication system. Digital Mobile Radio (DMR) is one of a digital radio standard for PMR developed by the European Telecommunications Standards Institute (ETSI) and published in 2006 [1]. DMR sets out a digital radio specification for professional, commercial and private radio users. DMR can be categorized into two types as conventional and trunked radio systems. In the conventional system, a frequency band is permanently dedicated to a voice transmission. In the trunked system, a communication link is allocated for the duration of a call and then automatically released to allow it to be used for another call, between different users on the same system [2].

Cell selection is the process of deciding the base station (BS) which provides services to each user. There are many studies for performing cell selection and user assignment for cellular wireless networks. In [3], a cell selection algorithm has been performed by utilizing proportional fair scheduling algorithm to assist the user by selecting the best serving cell while achieving highest achievable data rate. In [4], in order to achieve proportional fairness for all users in BS, a

\footnotetext{
${ }^{1}$ This work has been supported by Republic of Turkey Ministry of Science, Industry and Technology under SAN-TEZ 0686.STZ.2014 Programme.
}

cell selection has been formulated into a network-wide utility maximization problem. In [5], a cell selection algorithm has been examined for mobile networks with backhaul capacity constraints. This model analyzes the possibility to exploit load balancing among BSs depending on backhaul capacity utilization. Considering classical cell selection algorithm that user is assigned to the BS with maximum received power that causes unequal cell association in heterogeneous networks because of large difference in transmission power between macro cells and small cells. As a solution for this problem, the importance of considering both load balancing and interference management in cell selection process has been examined to achieve the throughput gain of multi-tiering networks in [6].

Load balancing is achieved by transferring users' traffic from macro cells to small cells. In two cell selection algorithms which uses in Long Term Evaluation (LTE) system maximize the network throughput: In Reference Signal Received Power (RSRP) based cell selection algorithm, the BS with maximum received power of reference signal is selected. In Reference Signal Received Quality (RSRQ) based algorithm that a BS is selected which maximize the RSRQ metric. In cell range expansion (CRE) approach in [7], the received power is biased by multiplying a bias value which is adjusted depending of the tier then the BS with maximum biased power is selected for connection. Since the smaller tier has greater bias value, this approach pushes the users toward lightly loaded BSs. However, optimization of bias values is needed for achieving the desired system utility. In path loss (PL) based cell selection, user selects the cell with the minimum path loss. Two different biased cell selection techniques to provide the desired throughput gains for users have been stated in [8]. In the first method, a serving cell is selected based on maximizing received signal strength with bias. Other method chooses a serving cell based on maximizing the product of signal-to-interference-noise ratio (SINR) and bias. In [9], the efficient network selection scheme has been given by using the positioning information and the map information. Using these information, a user selects a network based on the expected throughput and its location.

All these cell selection algorithms ignore the cell load and they are generally applied for heterogeneous networks to balance the load of macro and small cells. Because of 
their complexity and high overhead, all these cell selection techniques can not be directly applied for DMR systems which are classified as a single tier network.

In this paper, we propose an efficient cell selection algorithm for DMR tier II conventional to balance the users among the BSs. As a result, it is possible to reduce the waiting time to establish a connection without sacrificing bit-errorrate (BER) performance to establish a reliable transmission link. We compare the performance results with classical cell selection algorithms in terms of average BER and the cell load which is proportional to the total number of users associated to the each BS.

The rest of this paper is organized as follows. Section II describes the system model. Section III introduces the proposed cell selection algorithm. Section IV examines simulation parameters and performance results. Finally, section V gives the conclusions.

\section{SySTEM MODEL}

We consider a DMR system that consists of $U$ BSs and the total number of $Z$ users in the whole area. The DMR system with $7 \mathrm{BSs}$ is illustrated in Figure 1 in which the same color represents the same frequency. It is assumed that the users are distributed randomly in the considered area.

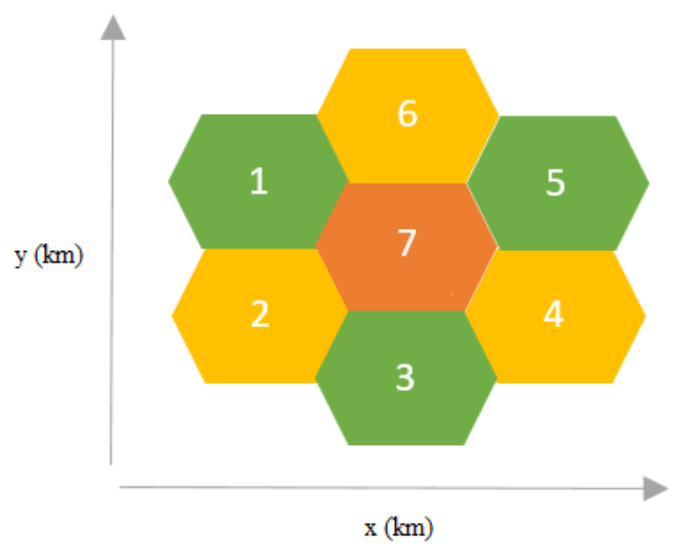

Fig. 1: An example of cell planning for DMR system

Audio and data transmissions are managed by two Time Division Multiple Access (TDMA) timeslots sharing the same $12.5 \mathrm{kHz}$ channel in a DMR system [10]. As a modulation scheme, 4FSK is used to generate at a rate of 4800 symbols/s, corresponding to $9600 \mathrm{bits} / \mathrm{s}$ [11].

For DMR system, the total number of available channels, $M_{u}$, belonging to $u$ th $\mathrm{BS}$ is determined as:

$$
M_{u}=2 \frac{B_{u}}{\Delta f}
$$

where $B_{u}$ is the available bandwidth for BS $u$ and $\Delta f$ is channel spacing. One of $M_{u}$ channels is dedicated for control and all the remaining ones are available for data communications [12].
For DMR systems, the common cell selection method is the one based on Received Signal Strength Indicator (RSSI). In RSSI based cell selection, each user selects the BS which has the maximum RSSI value as:

$$
u^{\prime}=\underset{1 \leq u \leq U}{\arg \max } R S S I_{u, k} \quad \forall k
$$

where $R S S I_{u, k}$ is the RSSI value belonging to $u$ th $\mathrm{BS}$ for user $k$ is determined by,

$R S S I_{u, k}=E I R P_{u}-P L_{u, k}-B u L_{k}-S h_{k}-B L+G_{r}-C L$

with $C L$ is receiver cable loss, $G_{r}$ is receiver antenna gain, $S h_{k}$ is shadowing modelled by $\log$ normal distribution, $B L$ is body loss, $B u L_{k}$ is building loss when the user $k$ is physically in the building, $P L_{u, k}$ is path loss between BS $u$ and user $k$ and $E I R P_{u}$ is the Effective isotropic radiated power (EIRP) for $\mathrm{BS} u$ which is determined by,

$$
E I R P_{u}=P_{u}^{t}+G_{t}
$$

where $P_{u}^{t}$ is transmit power for BS $u$ and $G_{t}$ is transmitter antenna gain.

Besides RSSI based cell selection, it is possible to apply SINR based cell selection:

$$
u^{\prime \prime}=\underset{1 \leq u \leq U}{\arg \max } S I N R_{u, k} \quad \forall k
$$

where

$$
S I N R_{u, k}=\frac{P_{u, k}}{I N T+N}
$$

with $P_{u, k}$ is the received power for user $k$ from BS $u, I N T$ is the interference power caused by the other cells having the same frequency, $N$ is the noise power. Interference power can be determined by assuming that the cell planning is known at each user.

Both SINR and RSSI based cell selection algorithms are commonly used for DMR systems because of their simplicity. However, these cell selection algorithms do not taken into account the cell load while assigning users to the BSs. When the cell load is not balanced in DMR systems, the users have to wait in the queue since the number of available channels is limited. Therefore, we propose a cell selection algorithm by considering both cell load and SINR value.

\section{PROPOSED LOAD BASED CELL SELECTION ALGORITHM}

The flowchart of the proposed cell selection algorithm is given Figure 2. 


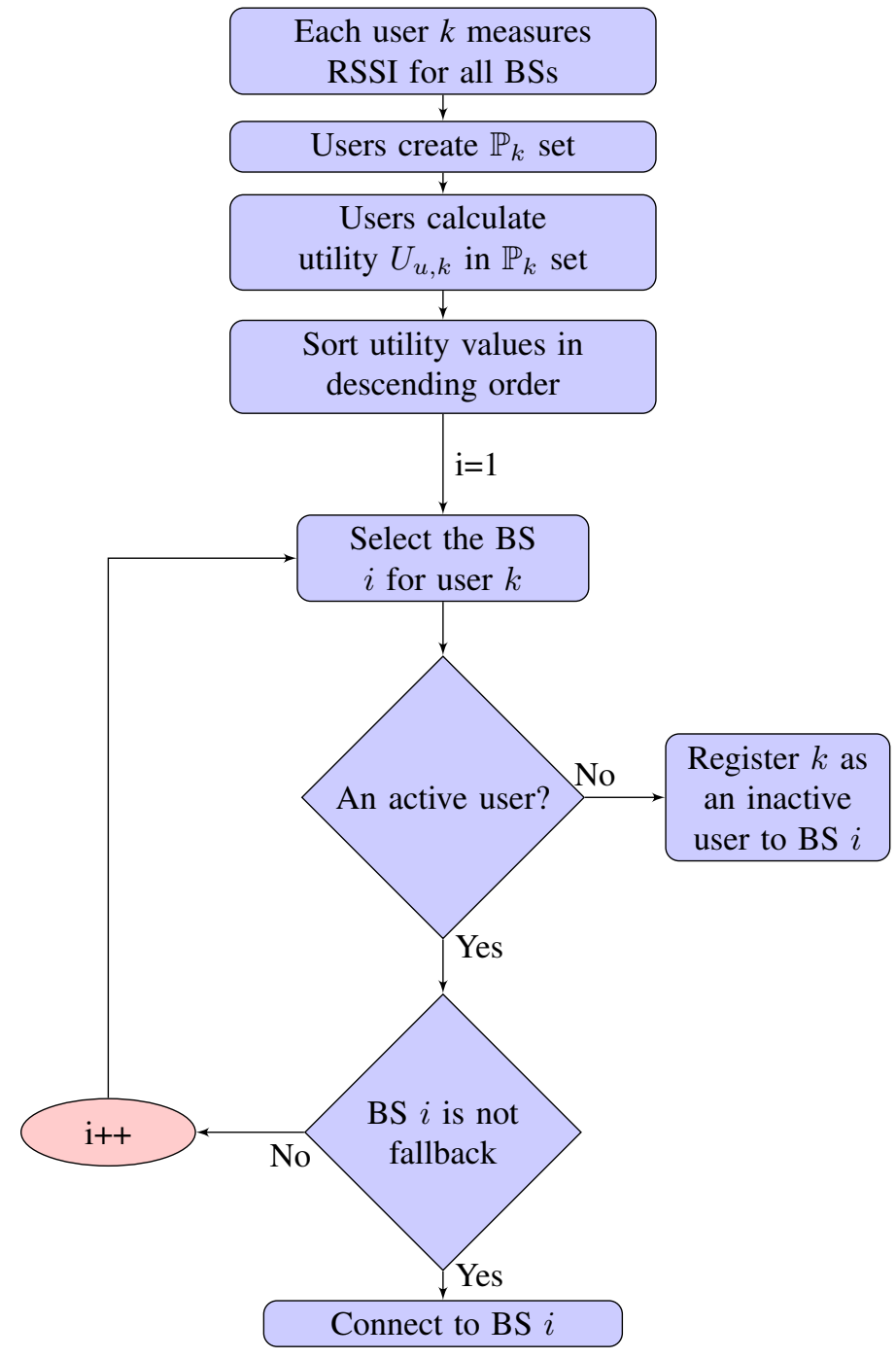

Fig. 2: Flowchart of Proposed Cell Selection Algorithm

- Firstly, each user constructs a set which satisfies a defined condition.

For user $k$, the received signal strength of BS $u$ should exceed a given predefined signal strength threshold, denoted by $R S S I_{t h}$ :

$$
\mathbb{P}_{k}=\left\{u \in U: R S S I_{u, k} \geq R S S I_{t h}\right\}
$$

- Then, each user calculates an utility value considering the BS in the constructed set.

For user $k$, the utility value is obtained by taking into account both SINR value and cell load parameter.

$U_{u, k}=w f\left(S I \bar{N} R_{u, k}+Q_{u}\right)+(1-w) g\left(U C L_{u}\right), \forall u \in \mathbb{P}_{k}$

where $w$ is the weight parameter between SINR and cell load, $Q_{u}$ is the given bias value for BS $u$, the function $f($.) represents the transformation of SINR values to the normalized SINR values and the function $g($.$) transforms$ the unmapped cell load (UCL) to the mapped one according to the predefined table. The $U C L_{u}$ is given by,

$$
U C L_{u}=(1-c) \frac{A_{u}}{M_{u}}+c \frac{I_{u}}{K_{u}}
$$

where $A_{u}$ and $I_{u}$ are respectively the number of active and inactive users attaching to $u$ th BS. The active users are attached to a BS and communicate whereas the inactive users are only attached but do not communicate. $c$ represents the importance of contribution of inactive users while determining the cell load. $M_{u}$ and $K_{u}$ represent the maximum possible number of active and inactive users in the cell respectively. $M_{u}$ is defined in Eq. (1) and $K_{u}$ is calculated by,

$$
K_{u}=N_{u}-M_{u}
$$

where $N_{u}$ determined by $Z / U$ is the total number of users per cell.

- For each user $k$, based on the utility value belonging to each BS in the set of $\mathbb{P}$, the BS which has the maximum utility value is selected as the target cell by,

$$
k_{u^{*}}=\underset{u \in \mathbb{P}_{k}}{\arg \max } U_{u, k}
$$

- If active users can not connect to any BS because of limited number of channels, they become a waiting user for its assigned BS.

In order to calculate the utility value at the user side for each BS, the $U C L_{u}$ value is critical importance. All related information including the number of active and inactive users, the total number of channels, the total number of base stations to obtain $U C L_{u}$ is available at BS $u$. Then, each BS calculates $U C L_{u}$, transforms it by using function $g($.$) according to given$ table. Finally, each BS broadcasts the corresponding index belonging its mapped value to all users at every predefined time slots.

\section{Performance Evaluations}

We perform simulation results considering $U=7 \mathrm{BSs}$ and $Z=700$ users in a metropolitan area as shown in Figure 3. The frequency reuse factor is taken as $1 / 3$. The transmit power of each BS is $50 \mathrm{dBm}$ [13]. The users are uniform distributed and $30 \%$ of users are assumed to be indoor environment.

There are only voice users that are required to allocate only one physical channel. Hata path loss model [14] is used for the urban area and indoor users experience extra building loss. The simulation parameters are given in Table I. 


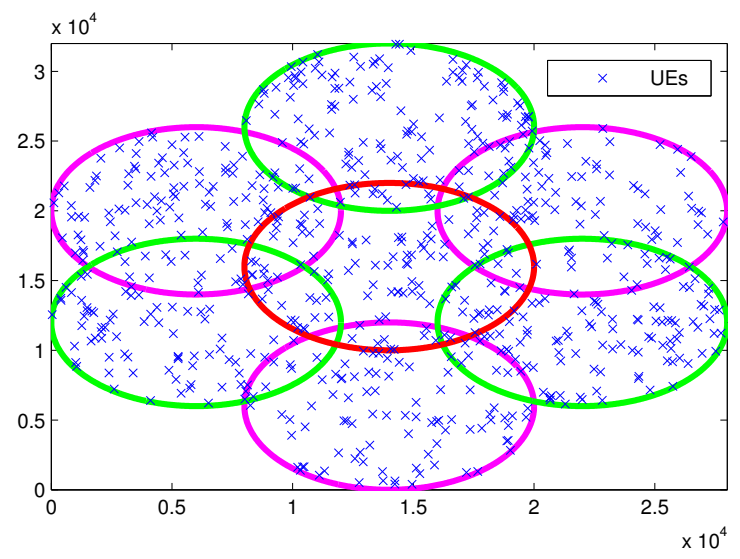

Fig. 3: Cell planning for DMR system

TABLE I: Simulation Parameters

\begin{tabular}{|c|c|}
\hline PARAMETERS & DMR \\
\hline Bandwidth per cell $(\mathrm{B})$ & $200 \mathrm{kHz}$ \\
\hline Modulation Bandwidth & $10 \mathrm{kHz}$ \\
\hline Channel Spacing $(\Delta f)$ & $12.5 \mathrm{kHz}$ \\
\hline Noise Spectral Density & $-174 \mathrm{dBm} / \mathrm{Hz}$ \\
\hline Receiver Sensitivity & $-110 \mathrm{dBm}$ \\
\hline Carrier frequencies & $415 \mathrm{MHz}$ \\
\hline Cell Radius & $6 \mathrm{~km}$ \\
\hline TX Antenna Gain & $8 \mathrm{~dB}$ \\
\hline RX Antenna Gain & $-2 \mathrm{dBi}$ \\
\hline RX Cable Loss & $2 \mathrm{~dB}$ \\
\hline BS Antenna Height & $30 \mathrm{~m}$ \\
\hline MS Antenna Height & $1.5 \mathrm{~m}$ \\
\hline Building Loss $[15]$ & $16.5 \mathrm{~dB}$ \\
\hline Body Loss & $10 \mathrm{~dB}$ \\
\hline Weight, $w$ & $0.3,0.5$ \\
\hline Coefficient, c & 0.1 \\
\hline Total Urban Area & $28 \mathrm{~km} \mathrm{x} 32 \mathrm{~km}$ \\
\hline
\end{tabular}

The percentage of active users that determines the traffic in the cell is listed in Table II. In this paper, the medium and high traffic cases are considered in the performance evaluations.

TABLE II: Traffic Cases

\begin{tabular}{|c|c|}
\hline Percentage of Active Users & Traffic Case \\
\hline $18 \%$ & Low \\
\hline $24 \%$ & Medium \\
\hline $31 \%$ & High \\
\hline
\end{tabular}

While calculating the utility value, the mapping is applied based on the predefined $f($.$) and g($.$) functions. Firstly, SINR$ values belonging to all BSs are sorted in descending order. Then, all SINR values including bias are assigned to the normalized values proportionally to their sorted SINR indexes given in Table III. Since one of the aim is that user connects the BS having the highest SINR value, the maximum SINR represent with the highest normalized value.

TABLE III: Normalization of SINR values with $f($.$) function$

\begin{tabular}{|c|c|}
\hline Index for ranked SINR & $\mathbf{f}\left(\mathbf{S I N R}+\mathbf{Q}_{\mathbf{u}}\right)$ \\
\hline 1 & 1 \\
\hline 2 & 0.8571 \\
\hline 3 & 0.7143 \\
\hline 4 & 0.5714 \\
\hline 5 & 0.4286 \\
\hline 6 & 0.2857 \\
\hline 7 & 0.1429 \\
\hline
\end{tabular}

The function $g($.$) calculates the cell load parameter that$ is modeled non-linearly to emphasis the high traffic behavior properly as given in Table IV. Since our target is to connect user to BS with low cell load, BS with highest UCL value which corresponds to the highest number of attached users is mapped to the lowest value. Based on the calculated UCL values in Eq. (9), the corresponding cell load indexes are assigned and then, each BS broadcasts its cell load at every predefined time slots by using 3 bits.

TABLE IV: Mapping of cell load values with $g($.$) function$

\begin{tabular}{|c|c|c|}
\hline Interval for UCL values & $\mathrm{g}(\mathbf{U C L})$ & Index \\
\hline $0-0.6$ & 1 & 1 \\
\hline $0.6-0.7$ & 0.86 & 2 \\
\hline $0.7-0.8$ & 0.71 & 3 \\
\hline $0.8-0.85$ & 0.57 & 4 \\
\hline $0.85-0.9$ & 0.43 & 5 \\
\hline $0.9-0.95$ & 0.29 & 6 \\
\hline $0.95-1$ & 0.14 & 7 \\
\hline
\end{tabular}

For the considered cell planning given in Figure 3, the BS labeled as 7 has not receive any interference and its SINR is relatively high compared to other BSs. Therefore, we apply $Q_{7}=-15 \mathrm{~dB}$ bias for BS labeled as 7 and $0 \mathrm{~dB}$ bias for the other BSs. Then, we obtain the simulation results for the proposed cell selection algorithm as unbiased and biased by considering different weights $(w)$ and different traffic cases. 


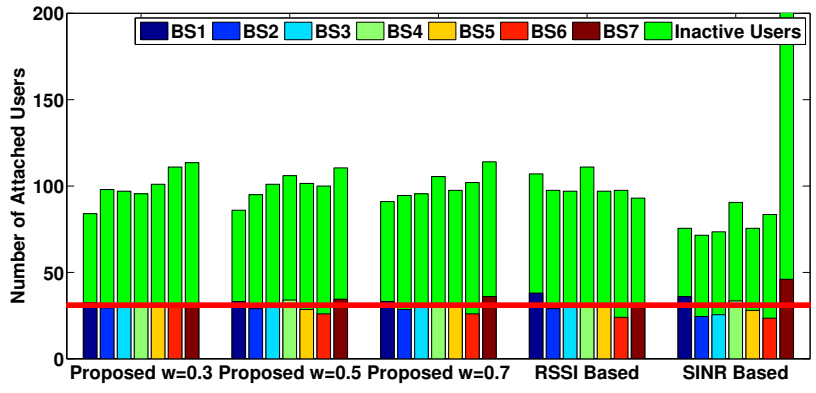

Fig. 4: Distribution of users among BSs in high traffic case without bias

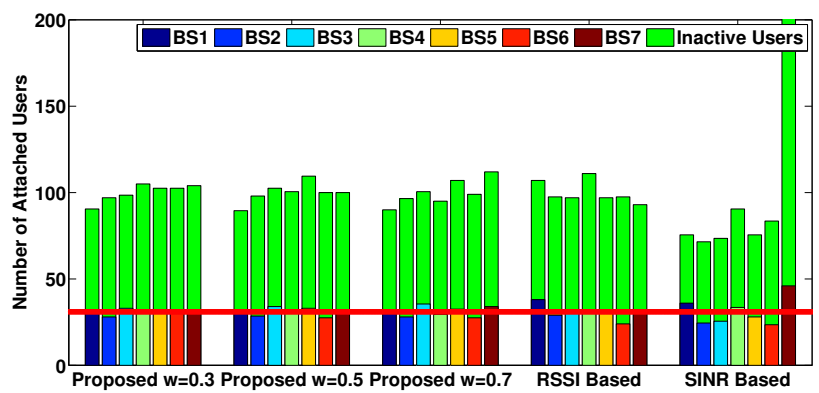

Fig. 5: Distribution of users among BSs in high traffic case with bias

We use different performance metrics as average delay counter, load fairness index and the number of waiting active users. The average delay counter is increased at each times when the user tries to connect any BS. Load fairness index is calculated to evaluate the fairness of the users' association among BSs by

$$
J I=\frac{Z^{2}}{U \sum_{j=1}^{U}\left(A_{u}^{2}+I_{u}^{2}\right)}
$$

The higher load fairness index represents a higher balanced among BSs. Waiting user is raised when an active user can not connect to any BS due to high cell load.

According to the simulation results illustrated in Figure 4 and Figure 5, it can be seen that the distribution of active and inactive users for the proposed cell selection algorithm is much more balanced compared to the other algorithms. Moreover, it is shown that the proposed algorithm with bias has a positive impact on balancing the distribution of UEs among all BSs. Considering cell load performance, the proposed algorithm with $w=0.3$ provides fairer distribution than the proposed algorithm with $w=0.5$ and $w=0.7$. The reason is that when $w$ is 0.3 , cell load becomes more important than SINR as given in Eq. (8). Since the BS labeled as 7 has received no interference from other BSs, most of the users are intended to connect it when SINR algorithm is employed.

Table V and Table VI show the performance results for the proposed algorithm with unbiased and biased, respectively. It is shown that the proposed algorithm with bias reduces the number of waiting active users compared to unbiased one. Since the proposed algorithm with $w=0.9$ does not consider cell load indicator, it gives the similar performance as SINR based algorithm.

TABLE V: Performance results without bias for high traffic case

\begin{tabular}{|c|c|c|c|}
\hline Algorithms & $\begin{array}{c}\text { Average } \\
\text { Delay } \\
\text { Counter }\end{array}$ & $\begin{array}{c}\text { Load } \\
\text { Fairness } \\
\text { Index }\end{array}$ & $\begin{array}{c}\text { Number } \\
\text { of Waiting } \\
\text { Active Users }\end{array}$ \\
\hline \hline $\begin{array}{c}\text { Proposed } \\
\text { w=0.1 }\end{array}$ & 1 & 0.997 & 38.6 \\
\hline $\begin{array}{c}\text { Proposed } \\
\text { w=0.3 }\end{array}$ & 1 & 0.995 & 38.6 \\
\hline $\begin{array}{c}\text { Proposed } \\
\text { w=0.5 }\end{array}$ & 1 & 0.996 & 38.6 \\
\hline $\begin{array}{c}\text { Proposed } \\
\text { w=0.7 }\end{array}$ & 1.0069 & 0.986 & 39.1 \\
\hline $\begin{array}{c}\text { Proposed } \\
\text { w=0.9 }\end{array}$ & 1.191 & 0.840 & 41.7 \\
\hline RSSI Based & 2.2475 & 0.988 & 39.3 \\
\hline SINR Based & 1.5362 & 0.809 & 41.7 \\
\hline
\end{tabular}

TABLE VI: Performance results with bias for high traffic case

\begin{tabular}{|c|c|c|c|}
\hline Algorithms & $\begin{array}{c}\text { Average } \\
\text { Delay } \\
\text { Counter }\end{array}$ & $\begin{array}{c}\text { Load } \\
\text { Fairness } \\
\text { Index }\end{array}$ & $\begin{array}{c}\text { Number of } \\
\text { Waiting } \\
\text { Active Users }\end{array}$ \\
\hline \hline $\begin{array}{c}\text { Proposed } \\
\text { w=0.1 }\end{array}$ & 1 & 0.994 & 35.4 \\
\hline $\begin{array}{c}\text { Proposed } \\
\text { w=0.3 }\end{array}$ & 1 & 0.991 & 35.4 \\
\hline $\begin{array}{c}\text { Proposed } \\
\text { w=0.5 }\end{array}$ & 1.0014 & 0.993 & 35.7 \\
\hline $\begin{array}{c}\text { Proposed } \\
\text { w=0.7 }\end{array}$ & 1.0108 & 0.980 & 36.2 \\
\hline $\begin{array}{c}\text { Proposed } \\
\text { w=0.9 }\end{array}$ & 1.2058 & 0.803 & 38.6 \\
\hline RSSI Based & 2.2475 & 0.988 & 39.3 \\
\hline SINR Based & 1.5362 & 0.809 & 41.7 \\
\hline
\end{tabular}

Although the high traffic case is much more critical, it is also proven that the same outcome is obtained for medium traffic case as Table VII. According to simulation results, the proposed algorithm with bias gives the best performance for medium traffic cases in terms of average delay counter, load fairness index and number of waiting active users compared to other algorithms.

In addition to that, we provide BER performance of the cell selection algorithms under fading channels as seen in Table VIII and Table IX for high and medium traffic cases, respectively. In order to guarantee service quality for DMR systems, BER must be under 0.05 for voice users. It is observed that the proposed algorithms achieve almost the same BER performance than SINR based algorithm while balancing the active and inactive users among the BSs to reduce the transmission delay. 
TABLE VII: Performance results with bias for medium traffic case

\begin{tabular}{|c|c|c|c|}
\hline Algorithms & $\begin{array}{c}\text { Average } \\
\text { Delay } \\
\text { Counter }\end{array}$ & $\begin{array}{c}\text { Load } \\
\text { Fairness } \\
\text { Index }\end{array}$ & $\begin{array}{c}\text { Number of } \\
\text { Waiting } \\
\text { Active Users }\end{array}$ \\
\hline \hline $\begin{array}{c}\text { Proposed } \\
\text { w=0.1 }\end{array}$ & 1 & 0.989 & 26.5 \\
\hline $\begin{array}{c}\text { Proposed } \\
\text { w=0.3 }\end{array}$ & 1 & 0.986 & 26.5 \\
\hline $\begin{array}{c}\text { Proposed } \\
\text { w=0.5 }\end{array}$ & 1 & 0.984 & 26.5 \\
\hline $\begin{array}{c}\text { Proposed } \\
\text { w=0.7 }\end{array}$ & 1 & 0.979 & 26.5 \\
\hline $\begin{array}{c}\text { Proposed } \\
\text { w=0.9 }\end{array}$ & 1.0089 & 0.959 & 26.5 \\
\hline RSSI Based & 2.1786 & 0.989 & 29.5 \\
\hline SINR Based & 1.4688 & 0.761 & 30.5 \\
\hline
\end{tabular}

TABLE VIII: BER performance for high traffic case

\begin{tabular}{|c|c|c|}
\hline Algorithms & $\begin{array}{c}\text { Outage } \\
\text { Probability } \\
\text { without Bias }\end{array}$ & $\begin{array}{c}\text { Outage } \\
\text { Probability } \\
\text { with Bias }\end{array}$ \\
\hline $\begin{array}{c}\text { Proposed } \\
\text { w=0.1 }\end{array}$ & 0.021 & 0.039 \\
\hline $\begin{array}{c}\text { Proposed } \\
\text { w=0.3 }\end{array}$ & 0.013 & 0.033 \\
\hline $\begin{array}{c}\text { Proposed } \\
\text { w=0.5 }\end{array}$ & 0.014 & 0.029 \\
\hline $\begin{array}{c}\text { Proposed } \\
\text { w=0.7 }\end{array}$ & 0.012 & 0.028 \\
\hline $\begin{array}{c}\text { Proposed } \\
\text { w=0.9 }\end{array}$ & 0.011 & 0.029 \\
\hline RSSI Based & \multicolumn{2}{|c|}{0.010} \\
\hline SINR Based & \multicolumn{2}{|c|}{0.018} \\
\hline
\end{tabular}

TABLE IX: BER performance for medium traffic case

\begin{tabular}{|c|c|c|}
\hline Algorithms & $\begin{array}{c}\text { Outage } \\
\text { Probability } \\
\text { without Bias }\end{array}$ & $\begin{array}{c}\text { Outage } \\
\text { Probability } \\
\text { with Bias }\end{array}$ \\
\hline \hline $\begin{array}{c}\text { Proposed } \\
\text { w=0.1 }\end{array}$ & 0.019 & 0.034 \\
\hline $\begin{array}{c}\text { Proposed } \\
\text { w=0.3 }\end{array}$ & 0.014 & 0.023 \\
\hline $\begin{array}{c}\text { Proposed } \\
\text { w=0.5 }\end{array}$ & 0.013 & 0.016 \\
\hline $\begin{array}{c}\text { Proposed } \\
\text { w=0.7 }\end{array}$ & 0.011 & 0.012 \\
\hline $\begin{array}{c}\text { Proposed } \\
\text { w=0.9 }\end{array}$ & 0.010 & 0.012 \\
\hline RSSI Based & \multicolumn{2}{|c|}{0.004} \\
\hline SINR Based & \multicolumn{2}{|c|}{0.010} \\
\hline
\end{tabular}

\section{CONCLUSION}

We have proposed an efficient load based cell selection method for professional mobile radios and have evaluated its performance considering DMR systems in metropolitan area considering different traffic loads. The users select the BS having the best utility value which takes into account both SINR and cell load information. The performance results have shown that the proposed algorithm with bias gives better performance than SINR and RSSI based cell selection methods in a manner of distribution of active and inactive users over all BS without requiring additional overhead. As a result, the waiting time to establish a connection is reduced while satisfying the BER requirements for reliable voice transmission.

\section{REFERENCES}

[1] Tait Radio Communications, 'Technologies and Standards for Mobile Radio Communications Networks', February 2010.

[2] ETSI Technical Specification 102 361-4, V1.2.1 (2006-09); 'Electromagnetic Compatibility and Radio Spectrum Matters (ERM); Digital Mobile Radio (DMR) Systems Part 4: DMR Trunking Protocol', September, 2006.

[3] Mohamed A. AboulHassan, Essam A. Sourour, Shawki E. Shaaban, 'Novel Cell Selection Algorithm for Improving Average User's Effective Data Rate in LTE HetNets', in 2014 IEEE Symposium on Computers and Communications (ISCC), pp.1-6, June 2014, Funchal, Portugal.

[4] J. Wang, J. Liu, D. Wang, J. Pang, G. Shen, 'Optimized Fairness Cell Selection for 3GPP LTE-A Macro-Pico HetNets', in Vehicular Technology Conference (VTC Fall), IEEE, pp.1-5, September 2011, San Francisco, CA, USA.

[5] Juan J. Olmos, Ramon Ferr'us, and Hiram Galeana-Zapi'en, 'Analytical Modeling and Performance Evaluation of Cell Selection Algorithms for Mobile Networks with Backhaul Capacity Constraints', in IEEE Transactions on Wireless Communications, vol. 12, no. 12, pp.6011-6023, December 2013.

[6] M. Chinipardaz, M. Rasti, M. Nourhoseini, 'An Overview of Cell Association in Heterogeneous Network: Load Balancing and Interference Management Perspective', in 2014 7th International Symposium on Telecommunications (IST'2014), IEEE, pp.1250-1256, September 2014, Tehran, Iran.

[7] H. Jo, Y. Sang, P. Xia, and J. Andrews, 'Heterogeneous Cellular Networks with Flexible Cell Association: A Comprehensive Downlink SINR Analysis', in IEEE Transactions on Wireless Communications, vol. 11, no. 10, pp.3484-3495, October 2012.

[8] K. Balachandran, J. Kang, K. Karakayali and K. Rege 'Cell Selection with Downlink Resource Partitioning in Heterogeneous Networks', in 2011 IEEE International Conference on Communications Workshops (ICC), pp.1-6, June 2011, Kyoto, Japan.

[9] J. Kuboniwa, Y. Miyake, S. Kameda, A. Taira, H. Oguma, N. Suematsu, T. Takagi, and K. Tsubouchi, 'High Efficient Network Selection Scheme Using Location Information for Heterogeneous Wireless System', in Wireless Communications and Networking Conference Workshops (WCNCW), IEEE, pp.391-396, March 2015, New Orleans, LA.

[10] ETSI Technical Specification $102 \quad 361-2 \quad$ V2.2.1 (201307),'Electromagnetic Compatibility and Radio Spectrum Matters (ERM); Digital Mobile Radio (DMR) Systems; Part 2: DMR Voice and Generic Services and Facilities', July 2013.

[11] ETSI Technical Specification 102 361-1 V2.2.1 (2013-02), 'Electromagnetic Compatibility and Radio Spectrum Matters (ERM); Digital Mobile Radio (DMR) Systems; Part 1: DMR Air Interface (AI) protocol', February 2013.

[12] ETSI Technical Specification 102 361-1 V1.4.5 (2007-12), 'Electromagnetic Compatibility and Radio Spectrum Matters (ERM); Digital Mobile Radio (DMR) Systems; Part 1: DMR Air Interface (AI) protocol', December 2007.

[13] Hans-Peter Ketterling, 'Introduction to Digital Professional Mobile Radio', Artech House, January 2004.

[14] ETSI Technical Report 143030 V9.0.0 (2010-02), 'Digital Cellular Telecommunications System (Phase 2+); Radio Network Planning Aspects', 3GPP TR 43.030 version 9.0.0 Release 9, February 2010.

[15] TIA Telecommunications Systems Bulletin TSB-88-B, 'Wireless Communications Systems, Performance in Noise and Interference, Limited Situations, Recommended Methods for Technology, Independent Modeling, Simulation, and Verifications', September 2004. 